Original Article

\title{
Effects of thoracic posture correction exercises on scapular position
}

\author{
WON-GYU YOO ${ }^{1)}$ \\ 1) Department of Physical Therapy, College of Healthcare Medical Science and Engineering, Inje Uni- \\ versity: 607 Obangdong, Gimhae, Gyeongsangnam-do 621-749, Republic of Korea
}

\begin{abstract}
Purpose] The purpose of this study was to investigate the effects of thoracic extension exercise on scapular alignment and thoracic kyphosis angle. [Subjects and Methods] Ten subjects with a kyphosis angle $\geq 40^{\circ}$ and aged between 20 and 26 years participated in this study. This study investigated the scapular forward distance and thoracic kyphosis angle before and after the thoracic extension exercise. [Results] The kyphosis angle after exercise was significantly decreased compared with that before exercise. Scapular forward distance after exercise significantly decreased compared to that before exercise. [Conclusion] The results of the present study suggest that correction of thoracic kyphosis should be included among exercises designed to achieve normal scapular alignment. Key words: Kyphosis angle, Scapular alignment, Thoracic kyphosis
\end{abstract}

(This article was submitted Nov. 13, 2017, and was accepted Dec. 12, 2017)

\section{INTRODUCTION}

The thoracic spine has natural kyphotic curves of about $20-40^{\circ 1)}$. However, excessive kyphotic curvature, as seen in the disease kyphosis, reflects structural deformation of the spine or a reduction in spinal structure due to incorrect posture in modern humans ${ }^{2}$. From a clinical perspective, higher loads of the spine is associated with a forward position of the head due to contraction of the levator scapular and upper trapezius muscles and weakness in the deep neck muscles ${ }^{2)}$. This, in turn, causes hyperextension of the upper cervical vertebrae, resulting in neck pain and tension headache, which may be acute or chronic ${ }^{3}$.

In normal scapular alignment, the scapular borders are about 2.5-3 inches from, and parallel to, the spine, and the scapula is held flat against the thorax between $\mathrm{T} 2$ and $\mathrm{T} 7$ at an angle of $5^{\circ}$ to the frontal plane ${ }^{3)}$. Kyphosis increases tension in the shoulder muscles, including the pectoralis major, subclavius, and pectoralis minor, and the shoulders show excessive internal rotation. Also, the rotator cuff muscles responsible for stability are weakened ${ }^{1,2)}$. As a result, the scapular alignment of individuals with kyphosis may not be normal ${ }^{4}$. Correct alignment of the thoracic spine can positively affect scapular positioning and alleviate muscle imbalance ${ }^{4}$. A variety of studies on thoracic extension exercises for kyphosis have been conducted, but studies of the impact on scapular alignment are insufficient. Therefore, this study investigated the effects of thoracic extension exercise on scapular alignment and thoracic kyphosis angle.

\section{SUBJECTS AND METHODS}

Ten subjects with a kyphosis angle $\geq 40^{\circ}$ and aged between 20 and 26 years (mean age: $22.2 \pm 1.7$ years; mean height: 170.4 $\pm 8.4 \mathrm{~cm}$; mean weight: $67.9 \pm 17.8 \mathrm{~kg}$ ) participated in this study. The purpose and methods of the study were explained to the subjects, and written informed consent was obtained in keeping with the ethical principles of the Declaration of Helsinki.

The PALM device (Performance Attainment Associates, St. Paul, MN, USA), which consists of an inclinometer and two caliper arms, was used to assess scapular forward distance. The bubble inclinometer is a semi-circular $\operatorname{arc}$ with $1^{\circ}$ gradations

Corresponding author. Won-gyu Yoo (E-mail: won7y@inje.ac.kr)

(C)2018 The Society of Physical Therapy Science. Published by IPEC Inc.

This is an open-access article distributed under the terms of the Creative Commons Attribution Non-Commercial No Derivatives (by-nc-nd) License. (CC-BY-NC-ND 4.0: https://creativecommons.org/licenses/by-nc-nd/4.0/) 
ranging from $0-30^{\circ}$ to each side of the midline. The examiner held the device with both hands, and the caliper tips were used to identify the pelvic bony landmarks. The degree of deviation from horizontal was read from the inclinometer. To compare the alignment of the scapulae before versus after the experiment, the scapular inferior angle and position relative to the spine were measured.

The thoracic kyphosis angle was measured using a dual digital inclinometer (Acumar; Lafayette Instrument, Lafayette, IN, USA). The first inclinometer tip was positioned at T1, and the second at T12. These spinal levels were determined by palpation. Measurements were made in a relaxed standing position with the subject adopting a natural posture.

For Exercise 1, the subject sat in a chair with his arms folded, while others sat around him looking down at his head. Five sets of five repetitions were performed with 1-minute intervals between sets. Exercise 2 began with neck, thorax, and back flexion. Then, the cervical and thoracic spine were divided into segments, and the target drew the shoulder blades at the end of the exercise. A total of five 5-second sets were performed, followed by three repetitions of a 10-second set for the neck and spine. These exercises were performed for a total of 12 days. This study investigated the scapular forward distance and thoracic kyphosis angle before and after the thoracic extension exercise.

Data were analyzed using SPSS for Windows software (ver. 24.0; SPSS Inc., Chicago, IL, USA). The paired t-test was used to compare the scapular forward distance and thoracic kyphosis angle before and after the thoracic extension exercise, and the significance level was set to $\alpha=0.05$.

\section{RESULTS}

The kyphosis angle after exercise $\left(41.60 \pm 6.60^{\circ}\right)$ was significantly decreased compared with that before exercise $(47.40$ $\left.\pm 3.50^{\circ}\right)(\mathrm{p}<0.05)$. Scapular forward distance after exercise $(7.86 \pm 1.74 \mathrm{~cm})$ significantly decreased compared to that before exercise $(8.40 \pm 1.78 \mathrm{~cm})$.

\section{DISCUSSION}

A study suggested that increased thoracic kyphosis may be associated with higher spinal loads and abnormal trunk muscle force in an upright stance ${ }^{1)}$. Bautmans et al. reported a 10-week calibration exercise for middle-aged women that resulted in a significant decrease in thoracic kyphosis, as well as an increase in the strength of the spinal muscles and positive changes in lung function ${ }^{5}$. The present study also found a significant decrease in the kyphosis angle after exercise. This decrease in the thoracic kyphosis angle resulted in re-positioning of the shoulders. Scapular forward distance was significantly decreased after the exercise protocol. When scapular alignment changes, the length of the scapular muscles also changes; normal scapular alignment is considered a factor affecting the shoulder muscle near the scapula ${ }^{3)}$. A previous study suggested that abnormal scapular alignment was related to scapular forward distance ${ }^{4}$. Ludewig et al. ${ }^{6}$ ) reported that scapular kinematic alterations similar to those found in patient populations have been identified in subjects with a short rest length of the pectoralis minor, tight soft-tissue structures in the posterior shoulder region, excessive thoracic kyphosis, or with flexed thoracic postures. This suggests that attention to these factors is warranted in the clinical evaluation and treatment of these patients. Thoracic extension exercises can strengthen the thoracic muscles, including the middle and lower trapezius. Correction of thoracic kyphotic posture could have a positive effect on scapular alignment. Exercises for normal scapular alignment focus on strengthening the shoulder muscles. The results of the present study suggest that correction of thoracic kyphosis should be included among exercises designed to achieve normal scapular alignment.

\section{Funding}

This research was supported by Basic Science Research Program through the National Research Foundation of Korea (NRF) funded by the Ministry of Education (No. 2017R1D1A1B03035485).

\section{Conflict of interest}

None.

\section{REFERENCES}

1) Panjabi MM, Takata K, Goel V, et al.: Thoracic human vertebrae. Quantitative three-dimensional anatomy. Spine, 1991, 16: 888-901. [Medline] [CrossRef]

2) Sahrmann SA: Diagnosis and treatment of movement impairment syndromes. St. Louis: Mosby, 2002.

3) Kendall FP, McCreary EK, Provance PG, et al.: Muscles: testing and function with posture and pain, 5th ed. Baltimore: Williams \& Wilkins, 2005.

4) Park SY, Yoo WG: Effect of sustained typing work on changes in scapular position, pressure pain sensitivity and upper trapezius activity. J Occup Health, 2013, 55: 167-172. [Medline] [CrossRef]

5) Bautmans I, Van Arken J, Van Mackelenberg M, et al.: Rehabilitation using manual mobilization for thoracic kyphosis in elderly postmenopausal patients with osteoporosis. J Rehabil Med, 2010, 42: 129-135. [Medline] [CrossRef]

6) Ludewig PM, Reynolds JF: The association of scapular kinematics and glenohumeral joint pathologies. J Orthop Sports Phys Ther, 2009, 39: 90-104. [Medline] [CrossRef] 